\title{
Intraspecific interactions among oophagous tadpoles (Chirixalus eiffingeri: Rhacophoridae) living in bamboo stumps in Taiwan
}

\author{
Yeong-Choy Kam, ${ }^{1 *}$ Yahn-Jauh Su, ${ }^{2}$ Jin-Liang Liu ${ }^{1}$ and Yao-Sung Lin $^{2}$ \\ ${ }^{1}$ Department of Biology, National Changhua University of Education, Changhua 50058, Taiwan, R.O.C. \\ ${ }^{2}$ Department of Zoology, National Taiwan University, Taipei, Taiwan, R.O.C.
}

(Accepted 18 January 2001)

\begin{abstract}
We studied the intraspecific interactions among oophagous Chirixalus eiffingeri tadpoles that occupied the same water-filled bamboo stumps at Chitou, Taiwan. We monitored the growth of newly-hatched tadpoles in unoccupied and occupied bamboo stumps in the field where the latter contained large tadpoles that hatched from earlier clutches. The growth of the small, late-hatching tadpoles in occupied nests was suppressed by the presence of large, early-hatching tadpoles. However, small tadpoles that were physically separated from large tadpoles in a perforated container grew at about the same rate as small tadpoles living in pools without large tadpoles. Thus, the slower growth of the late-hatching tadpoles was probably caused by behavioural interference competition with the early-hatching tadpoles. In the laboratory, we kept large and small tadpoles together in containers and did not feed them for 6 days. The large tadpoles did not cannibalize the small tadpoles. Although large tadpoles may scavenge dead tadpoles, the effects of scavenging on growth were negligible. Interactions among cohabiting C. eiffingeri tadpoles are similar to those among the oophagous tadpoles of several hylid species that use phytotelmata. Results suggest that behavioural interference competition is the principal type of intraspecific interactions among the oophagous, non-predatory tadpoles of hylid and rhacophorid frogs.
\end{abstract}

Key words: Chirixalus eiffingeri, intraspecific interactions, oophagy, Taiwan

\section{INTRODUCTION}

Arboreal water pools, which may occur in tree holes and cavities, bamboo stumps, bromeliad leaf axils, nut capsules and others sites, are unique and isolated microhabitats (Laessle, 1961; Wassersug, Frogner \& Inger, 1981; Lannoo, Townsend \& Wassersug, 1987; Caldwell, 1993; Kam, Chuang \& Yen, 1996). For many organisms, arboreal pools serve as seasonal or permanent aquatic habitats in an otherwise terrestrial setting. Dipterans, helodid beetles and dragonflies are common in arboreal pools (Fish, 1983), as are other, non-insect invertebrates, including protozoans, flatworms, crustaceans, oligochaetes, ostracods, copepods and rotifers (Laessle, 1961; Fish, 1983). Anurans are the only vertebrates that use arboreal pools for breeding (Laessle, 1961; Duellman \& Trueb, 1986; Caldwell, 1993; Kam et al., 1996). However, their use of arboreal pools is not uniform and different species have evolved different reproductive modes (for details, see Duellman \& Trueb,

*All correspondence to: Yeong-Choy Kam.

E-mail: biyckam@cc.ncue.edu.tw
1986). For example, Dendrobates pumilio females lay eggs in terrestrial leaf litter, and then transport newly hatched tadpoles to water-filled leaf axils on their back (Brust, 1993). Other species lay their eggs above, or in, arboreal water pools, and the tadpoles drop into the pools as soon as they hatch (Lannoo et al., 1987; Caldwell, 1993; Jungfer, 1996, Kam et al., 1996)

Arboreal pools typically contain small volumes of stagnant, hypoxic water, with limited food resources. Trophic eggs (fertilized or unfertilized) are the most common food for tadpoles and are provided intermittently by genetically related female frogs (Weygoldt, 1980; Lannoo et al., 1987; Brust, 1993; Jungfer, 1996; Kam et al., 1996; Caldwell \& de Oliveira, 1999). The behaviour and life-history traits of frogs that breed in arboreal pools are diverse. Poisonous frogs in the genus Dendrobates have very small clutches (two to six eggs) and typically transport their tadpoles singly to small phytotelmata, such as bromeliad tanks or leaf axils. In contrast, hylid frogs in the genera Anotheca, Osteopilus and Osteocephalus have large clutches (in hundreds) and an entire clutch of tadpoles will live in a small, arboreal pool (Jungfer, 1996; Thompson, 1996; Jungfer \& Wey- 
goldt, 1999). Many species of tadpoles are predacious, consuming conspecifics (cannibalism) or heterospecifics (Caldwell, 1993; Caldwell \& de Araújo, 1999; Summers, 1999). In some, but not all, species there is communication between the mother and her tadpoles (Brust, 1993; Caldwell \& de Oliveira, 1999; Jungfer \& Weygoldt, 1999).

To date, most field research on the growth, and intraspecific and interspecific interactions between arboreal tadpoles has been conducted on various hylid and dendrobatid species in Central and South America. There has been much less research on arboreal tadpoles in the Old World. In the Oriental region, some species of Chirixalus and Philautus, family Rhacophoridae, are arboreal breeders whose tadpoles live in tree holes or bamboo stumps (Wassersug et al., 1981; Kam et al., 1996). Chirixalus eiffingeri breeds mainly in bamboo stumps in moist, montane areas in Taiwan (Kam et al., 1996). Female C. eiffingeri use only $40 \%$ of the available bamboo stumps as nest sites, and suitable stumps are reused as the breeding season progresses (Kam et al., 1996). It is not unusual for new egg clutches to be deposited in stumps already containing tadpoles, resulting in two age classes of tadpoles in a single pool. Relatively little is known about the interactions between the tadpoles in the two cohorts. Cannibalism should be common among C. eiffingeri tadpoles (Crump, 1992) because they usually live in extremely crowded conditions (nine tadpoles/100 $\mathrm{ml}$ or equivalent to 65506 tadpoles $/ \mathrm{m}^{3}$ ) (Kam et al., 1998a) and trophic eggs are available only sporadically and for brief periods of time.

In this study, we investigated the intraspecific interactions between $C$. eiffingeri tadpoles from different cohorts and assessed the prevalence of tadpole cannibalism among tadpoles.

\section{MATERIALS AND METHODS}

\section{Natural history of $C$. eiffingeri}

The rhacophorid tree frog, C. eiffingeri, is a small frog with a snout-vent length of $30-40 \mathrm{~mm}$. It is endemic to Taiwan and 2 nearby, small islands, Iriomote and Ishigaki (Kuramoto, 1973; Ueda, 1986; Lue, 1990). During the breeding season (February-August), female frogs deposit fertilized eggs on the inner walls of bamboo stumps or tree holes, just above the waterline (Kuramoto, 1973; Kam, Yen \& Hsu, 1998b). Male frogs exhibit paternal care during the embryonic stage, but they leave the stumps after the embryos have hatched. Upon hatching, tadpoles drop into the water pool where they grow and develop until metamorphosis. Tadpoles are obligatorily oophagous and are fed by females that, in the absence of male frogs, lay unfertilized trophic eggs directly in the water. Female frogs visit and feed tadpoles only at night, at intervals of about 8 days (Kam et al., 2000). The length of the tadpole period, from hatching to metamorphosis, is from 50 to 60 days (Kam et al., 1998a).

\section{Study site}

All experiments were conducted in bamboo forests at the Experimental Forest of National Taiwan University, Chitou (elevation $1016 \mathrm{~m}$, approximately $23^{\circ} 39^{\prime} 20^{\prime \prime} \mathrm{N}$, $120^{\circ} 48^{\prime} 10^{\prime \prime}$ E), Nantou County, Taiwan. Chitou receives approximately $3 \mathrm{~m}$ of rainfall annually. Although rain falls in all months, the wet season begins in February and ends in late September. The mean annual air temperature is about $17^{\circ} \mathrm{C}$.

\section{Experiment 1: growth and survivorship of $C$. eiffingeri tadpoles in unmanipulated, unoccupied and occupied nests}

We conducted this experiment from July to October 1994, in 2 bamboo (Phyllostachys edulis) groves of approximately $20 \times 35 \mathrm{~m}$ each. Near the end of the breeding season, in early August, we found 13 fertilized egg clutches above the water line on the inner wall of bamboo stumps. Fertilized eggs have distinct animal and vegetal hemispheres and embryos were usually developing when we found the eggs. In contrast, infertile eggs do not have animal and vegetal hemispheres and the egg yolk appears cloudy. In three bamboo stumps, eggs were laid above pools that already contained developing tadpoles. Tadpoles from earlier clutches are hereafter called EARLY tadpoles, and those from later clutches are hereafter called LATE tadpoles. EARLY tadpoles could be easily distinguished from LATE tadpoles by their larger body size and later developmental stage. The inner diameter of stumps and the water depth were measured to calculate tadpole density.

We monitored the growth and survivorship of all tadpoles for 4 weeks. To facilitate the examination and measurement of tadpoles, on our first visit we sawed a $\mathrm{V}$-shaped cut in the base of the bamboo stump and detached the portion of the stump containing the water and tadpoles. This allowed us to easily pour out the water and tadpoles. After data collection the water and tadpoles were placed back in the detached portion of the stump, which was then placed in the V-shaped notch in the stump base. The detached portion fitted snugly in the notch and transparent tape was wrapped around the cut for additional support. During each visit we poured the tadpoles and water into a bucket and used a small net to carefully transfer tadpoles to petri dishes. The tadpoles were counted and photographed; a ruler was placed beneath the petri dish to determine their total length (TL).

Because female frogs feed their tadpoles at night, we minimized disturbance of maternal feeding behaviour by examining the bamboo stumps during the day (Kam et al., 2000). Due to time constraints, we did not study the feeding behaviour of female frogs. Before tadpoles reached a TL of about $20 \mathrm{~mm}$, we recorded the presence of jelly capsules in the pools, which we took as evidence of tadpoles having been fed by the female frog. We did 
this because small tadpoles can only bite through the jelly capsule and suck out the yolk, leaving the jelly capsule in the pool (Ueda, 1986). As tadpoles grow larger, they usually ingest the whole egg, including the capsule.

\section{Experiment 2: indirect effects of large tadpoles on the growth and survivorship of small tadpoles}

We conducted this experiment from March to July 1998. To begin, we located bamboo stumps with fertilized eggs attached to the inner wall above the pool. We monitored the egg clutches twice a week and collected the tadpoles after they hatched. At the same time, we located bamboo stumps containing developing tadpoles and collected the tadpoles when they reached designated sizes. The small tadpoles were about 1 week old, $10-13 \mathrm{~mm}$ in TL, and at Gosner stage 25-26 at the start of the experiment (Gosner, 1960). The large tadpoles were about 5 weeks old, 26-28 mm long, and at Gosner stage 30-31.

We removed all the tadpoles from 32 bamboo stumps and then placed 10, randomly selected, newly hatched tadpoles into each of the 32 stumps. We designated a bamboo stump as an experimental unit, and each treatment group consisted of 16 randomly selected stumps. For the experimental group, we placed a perforated centrifuge tube $(50 \mathrm{ml}$; height $\times$ outer diameter: $105 \times 29 \mathrm{~mm}$ ) containing 5 large tadpoles into the pool in each of 16 bamboo stumps. For the control group, we put a perforated centrifuge tube with no tadpoles into the pool in each of the remaining 16 bamboo stumps. The centrifuge tubes were capped so that the large tadpoles could not receive trophic eggs from female frogs. Water could flow freely between the centrifuge tube and the pool in which each was immersed, allowing tadpole excrement, which consists of very fine suspended particles, and soluble chemicals to circulate freely (Y-C. Kam, per. obs.). We replaced the large tadpoles in the centrifuge tubes weekly with tadpoles of similar size so the large tadpoles did not starve. Fasting for 1 week was unlikely to cause any ill effect on the large tadpoles because, under natural conditions, C. eiffingeri tadpoles are fed by female frogs at intervals of about 8 days (Kam et al., 2000). Between feedings, trophic eggs stored in a tadpole's distensible stomach are the main source of energy (Chou \& Lin, 1997)

We maintained the water volume of each pool such that there were $10 \mathrm{ml}$ of water for each tadpole, regardless of tadpole size. For example, pools with 10 and 15 tadpoles contained 100 and $150 \mathrm{ml}$ of water, respectively. This was the average tadpole density we observed under natural conditions, as in Experiment 1. We added distilled water to compensate for water loss from evaporation and removed excess water from rainfall.

We monitored the growth and survivorship of small tadpoles for 4 weeks using the same protocol as in Experiment 1.

\section{Experiment 3: incidence of cannibalism among C. eiffingeri tadpoles}

We assessed the incidence of tadpole cannibalism using 3 treatments: 10 small tadpoles ( $\mathrm{S}$ treatment); 10 small and 10 large tadpoles (LS treatment); 10 large tadpoles ( $\mathrm{L}$ treatment). The small and large tadpoles were the same as in Experiment 2. All large tadpoles were fasted for at least 7 days until their stomachs were empty of stored, trophic eggs, which can be determined easily with the naked eye.

Each treatment had 5 replicates. In each replicate the tadpoles were kept in a plastic cup at a density of 1 tadpole per $10 \mathrm{ml}$ water, regardless of tadpole size. The number of surviving tadpoles in each replicate were counted each day for 6 days. The large tadpoles were weighed at the start and end of the 6 days of the experiment. Each tadpole was weighed separately. We tared a small petri dish containing water, added a tadpole, and recorded the body mass.

\section{Statistical analyses}

Data were analysed using a SAS program (SAS Institute, 1996). Each stump was an experimental unit. The treatment mean for all measured variables was calculated from the mean of the corresponding variable from each clutch. In Experiment 1, 2 clutches in the 10 unoccupied nests were not fed by female frogs. In Experiment 2, there was no evidence of maternal feeding of 5 of 16 clutches in both the control group and the experimental group. Clutches of unfed tadpoles were excluded from statistical analyses. We used analysis of covariance (ANCOVA) to analyse tadpole growth, with initial TL as a covariate. We used repeated measures ANOVA to analyse tadpole survivorship. We used $t$-tests or Mann-Whitney tests to compare the means or mediums of 2 samples, respectively. For all variables means \pm SE are reported, unless otherwise noted.

\section{RESULTS}

Experiment 1 was aimed to monitor the growth and survivorship of tadpoles in unoccupied and occupied nests. At the beginning of the experiment, EARLY tadpoles $(24.5 \pm 2.79 \mathrm{~mm}, n=3)$ in occupied nests were significantly larger than LATE tadpoles (12.57 $\pm 0.35 \mathrm{~mm}, n=3$; Mann-Whitney test, $Z=1.94$, $P=0.048)$. EARLY tadpoles maintained a size advantage over LATE tadpoles throughout the tadpole stage. EARLY and LATE tadpoles lived together for about 4 weeks until EARLY tadpoles reached metamorphosis.

Because stump hole diameter and water depth varied, we calculated the water volume and tadpole density. The tadpole density in unoccupied nests $(7.99 \pm 5.4$ tadpole/100 ml water, $n=8$ ) was not significantly different from that in occupied nests $(8.30 \pm 5.4$ tadpole/ $100 \mathrm{ml}$ water, $n=3$; Mann-Whitney test, $Z=0.102$, 


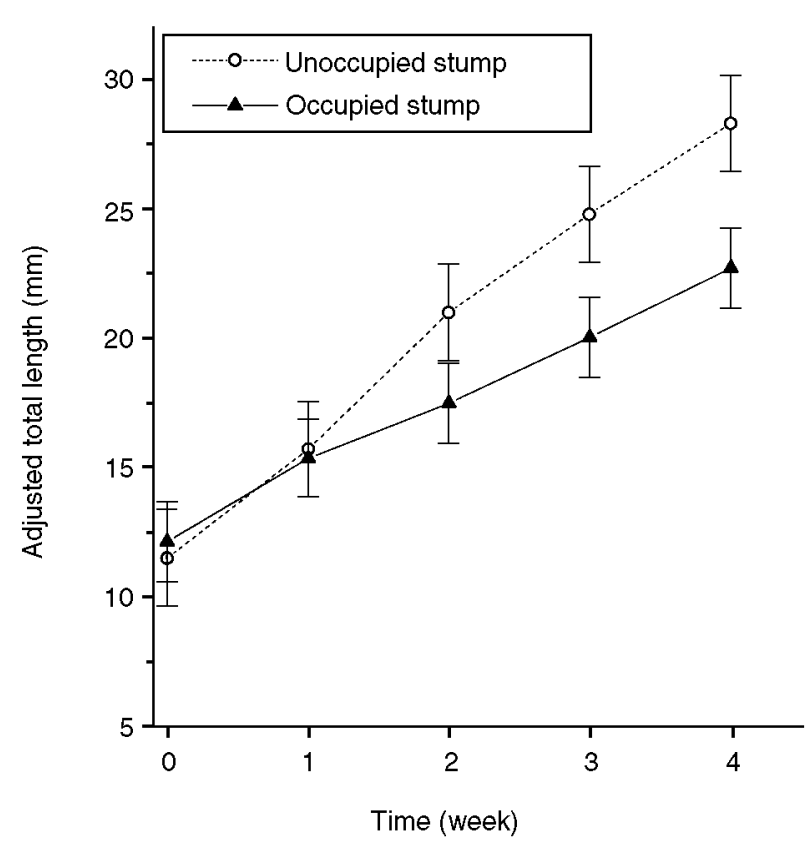

Fig. 1. Total length of small (late-hatching) tadpoles in occupied stumps and tadpoles in unoccupied stumps in Experiment 1. Total length of tadpole was adjusted by ANCOVA, and initial length was used as a covariate. Values are means \pm SE. Sample sizes are three and eight bamboo stumps for respective groups.

$P=0.918)$. The growth of LATE tadpoles in occupied nests was significantly slower than that of small tadpoles in unoccupied nests (ANCOVA, $F_{1,8}=5.85, P=0.042$; Fig. 1). In contrast, the survivorship of the tadpoles in these two treatments was not significantly different (repeated measure ANOVA, $F_{1,9}=0.68, P=0.432$ )

Experiment 2 was aimed to assess the indirect effects of large tadpoles on the growth and survivorship of small tadpoles. Results showed that the growth (ANCOVA: $F_{1,19}=0.03, P=0.8688$; Fig. $2 \mathrm{a}$ ) and survival (repeated measures ANOVA: $F_{1,20}=0.10$, $P=0.753$; Fig. 2 b) of small tadpoles in the control and experimental groups were not significantly different.

Experiment 3 was aimed to assess the prevalence of cannibalism among tadpoles. Throughout the study period, we have never observed large tadpoles attacking small tadpoles in the LS treatment. By the end of the experiment, the number of small tadpoles in the $\mathrm{S}$ and LS treatments had decreased from 10 to $8.4 \pm 0.9(n=5)$ and $9.0 \pm 0.7(n=5)$, respectively. These numbers were not significantly different $(t=1.177, P=0.273$ ).

All large tadpoles in the LS and L treatments survived to the end of the experiment, but they all lost weight. The mean initial body mass of large tadpoles in the LS treatment was $139.5 \pm 16.8 \mathrm{mg}(n=5)$, and they lost an average of $8.74 \pm 2.37 \mathrm{mg}$ during the experiment. The mean initial body mass of large tadpoles in the $\mathrm{L}$ treatment was $138.1 \pm 12.6 \mathrm{mg}(n=5)$, and they lost an average of $11.80 \pm 3.36 \mathrm{mg}$. There was no significant difference in the weight loss of large tadpoles in the LS and $\mathrm{L}$ treatments $(t=1.67, P=0.135)$.
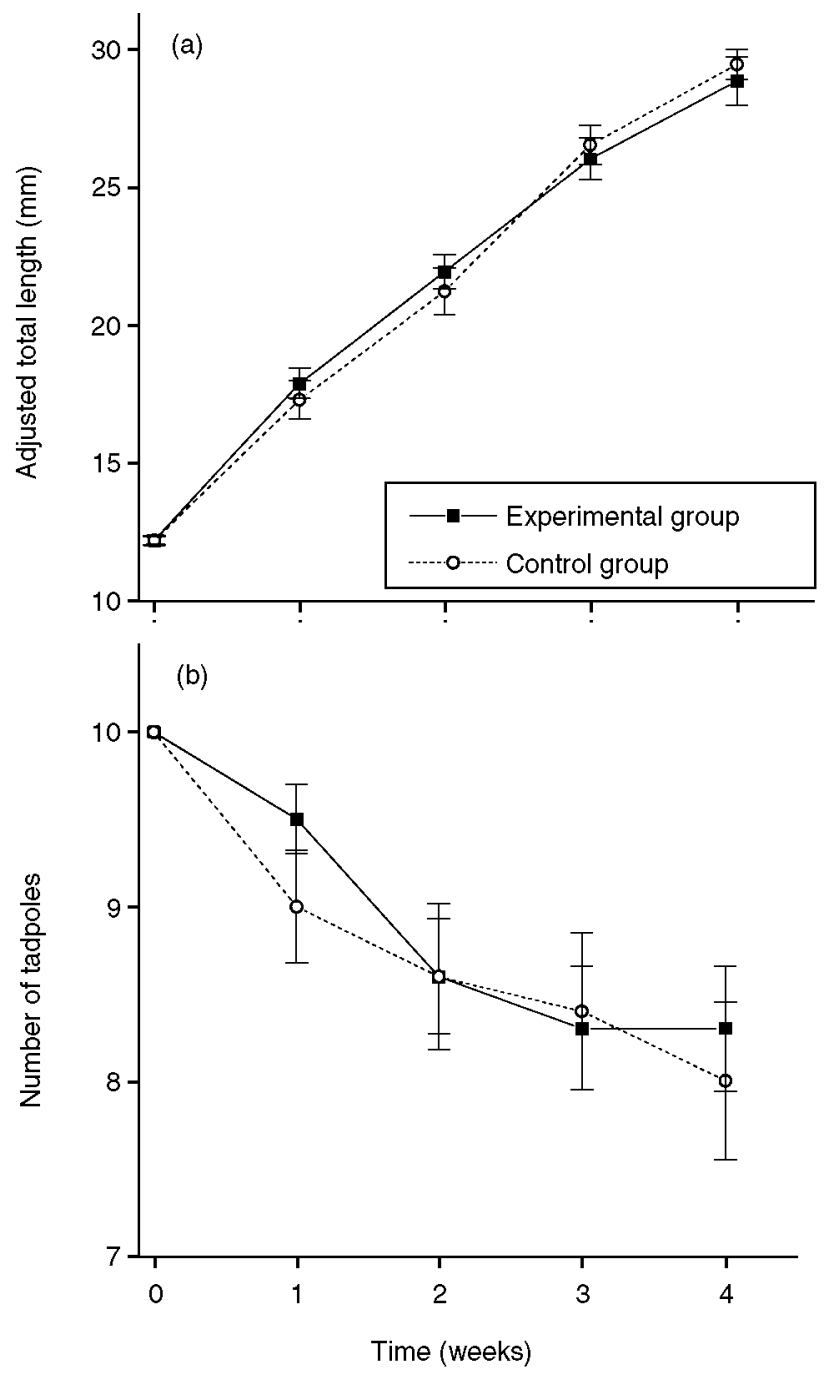

Fig. 2. The (a) total length and (b) number of surviving tadpoles during development in Experiment 2. The total length of tadpoles was adjusted by ANCOVA, and initial length was used as a covariate. Values are means \pm SE. Sample sizes are 11 bamboo stumps for each group.

\section{DISCUSSION}

In Experiment 1, in pools in which two clutches of tadpoles of different ages coexisted, tadpoles in the late hatching cohort (LATE tadpoles) grew slower than small tadpoles in pools lacking large tadpoles (Fig. 1). This indicates that tadpoles in early hatching cohorts (EARLY tadpoles) exert negative effects on the growth of LATE tadpoles. In Experiment 2, in which large tadpoles were physically separated from the small tadpoles in the same pool, the small tadpoles grew at the same rate as tadpoles in the control group (Fig. 2a,b). Elimination of the behavioural interactions between large and small tadpoles allowed for normal growth of the small tadpoles, suggesting that behavioural interference competition has probably caused the decreased growth of LATE $C$. eiffingeri tadpoles. Size- and agespecific competition has been used to explain disparities 
in the growth of other amphibian larvae (Savage, 1952; Wilbur, 1977; Semlitsch \& Caldwell, 1982). Chirixalus eiffingeri tadpoles are obligatorily oophagous and are fed intermittently by females that lay unfertilized, trophic eggs. When a female frog returns to the pool, tadpoles immediately aggregate around her. Tadpoles begin stiffing their tails, vibrating vigorously and nipping at the skin around her cloaca and thigh areas (Ueda, 1986). Without the presence of male frogs, the female then begins to lay trophic eggs, a few at a time. As soon as the eggs are laid, the tadpoles bite them and suck out the yolks in seconds (Ueda, 1986; Lin, 1996). EARLY tadpoles are bigger and stronger, and better able to fight for a position in the vicinity of the cloaca. In addition, their vigorous swimming movements may prevent LATE tadpoles from getting near the cloaca. We suggest that EARLY tadpoles outcompete LATE tadpoles by monopolizing most of the eggs laid by the female frog. As a result, LATE tadpoles were unable to obtain sufficient food and grow more slowly than conspecifics of the same age that do not have to compete with older tadpoles (Fig. 1).

From Experiment 2 we inferred that chemical interference is unimportant among $C$. eiffingeri tadpoles (Fig. 2a,b). If chemical interference competition among C. eiffingeri tadpoles were important, small tadpoles in the LS treatment, which lived in water conditioned by the large tadpoles, would have grown more slowly than tadpoles in the $\mathrm{S}$ treatment. However, the growth and survival of small tadpoles in the control and experimental groups were not significantly different (Fig. $2 a, b)$. To date, few experiments have assessed the incidence and importance of chemical interference competition in tadpole populations in natural habitats, under natural conditions. Petranka (1989) experimentally demonstrated that chemical interference competition among tadpoles of the southern leopard frog Rana utricularia is uncommon in natural habitats, even though it has been demonstrated in laboratory populations of this species (Steinwascher, 1978) and several, closely related congeners (Richards, 1958, 1962; Licht, 1967; Steinwascher, 1979a,b). Steinwascher (1978) found that water taken from local aggregates inhibited growth only when tadpoles densities were extremely high (14557 tadpoles $/ \mathrm{m}^{3}$ or two tadpoles/ $100 \mathrm{ml})$. In this study, there was no evidence for chemical interference competition among $C$. eiffingeri tadpoles, even at a density of nine tadpoles $/ 100 \mathrm{ml}$. It is possible that ambient $\mathrm{pH}$, exposure to UV radiation, consumption of, or competition with, micro-organisms by tadpoles prevent inhibitory substances, yeast cells in particular, from reaching concentrations sufficient to cause chemical interference (Petranka, 1989).

In Experiment 3, large tadpoles did not cannibalize conspecific tadpoles, although there was some tadpole mortality. Large tadpoles in the SL treatment lost as much weight as those in the L treatment. Large tadpoles did consume tadpoles that had died, but these were apparently insufficient to support the basic, energy requirements of the large tadpoles. We reached a similar conclusion based on the results of earlier field studies (Kam et al., 1998a; Kam et al., 2000). Under natural conditions, female $C$. eiffingeri abandon about $30 \%$ of the nests and the tadpoles do not receive trophic eggs. If scavenging were important, we would expect some tadpoles in abandoned nests to scavenge dead tadpoles, grow and maybe even reach metamorphosis. However, these studies showed that no orphaned tadpole increased in weight (Kam et al., 1998a, 2000). In addition, the other life forms (euglenoids, diatoms, parameciums, Chlorella, rotifers and dipteran larvae) in the pools do not appear to have a direct association with the tadpoles as they are neither food for nor predators of the tadpoles (Y.-C. Kam, pers. obs.).

Arboreal pools are restricted microhabitats, thus competition for space and food among the inhabitants may be severe. Many species of Dendrobates have evolved predatory behaviour to eliminate competitors or predators (Weygoldt, 1987; Caldwell \& de Araújo, 1999; Summers, 1999). Cannibalism is thought to be a secondary outcome of this predatory behaviour (Crump, 1992). In contrast to tadpoles of the Dendrobates histrionicus species group (Myers, Daly \& Martínez, 1984; Weygoldt, 1987; Brust, 1993), the hylid species Osteopilus brunneus, Osteocephalus oophagous and Anotheca spinosa (Jungfer, 1996; Thompson, 1996; Jungfer \& Weygoldt, 1999) and the rhacophorid C. eiffingeri are not predaceous. It is common to find many tadpoles of these species coexisting peacefully in arboreal pools. Jungfer (1996) reported that when many (> 10) A. spinosa tadpoles were present in a container, two distinct groups the members of which differed in size and developmental stage could soon be recognized. Those that regularly received nutritive eggs grew rapidly, while those that obtained few or no eggs had stunted growth. Observations were made in $O$. brunneus (Thompson, 1996) and C. eiffingeri (Kam et al., 1998b). Thus, large tadpoles apparently prevent small tadpoles from getting food through behavioural interference competition. As more of the starved tadpoles die, tadpole density in the pool is reduced.

\section{Acknowledgements}

This study was supported by a National Science Council Grant (NSC 89-2311-B-018-004) to YCK. We would like to thank the staff of the Experimental Forest of National Taiwan University at Chitou for providing accommodations and permitting us to work in the experimental forest. A. F. Warneke provided useful comments on an earlier draft of this manuscript.

\section{REFERENCES}

Brust, D. G. (1993). Maternal brood care by Dendrobates pumilio: a frog that feeds its young. J. Herpetol. 27: 96-98.

Caldwell, J. P. (1993). Brazil nut fruit capsules as phytotelmata: interactions among anuran and insect larvae. Can. J. Zool. 71: 1193-1201. 
Caldwell, J. P. \& de Araújo, M. C. (1999). Cannibalistic interactions resulting from indiscriminate predatory behaviour in tadpoles of poison frogs (Anura: Dendrobatidae). Biotropica 30: $92-103$.

Caldwell, J. P. \& de Oliveira, V. R. L. (1999). Determinants of biparental care in the spotted poison frog, Dendrobates vanzolinii (Anura: Dendrobatidae). Copeia 1999: 563-575.

Chou, W.-H. \& Lin, J.-Y. (1997). Tadpoles of Taiwan. Spec. Publ. No 7, Natl. Mus. Nat. Sci.

Crump, M. L. (1992). Cannibalism in amphibians. In Cannibalism: ecology and evolution among diverse taxa: 256-276. Elgar, M. A. \& Crespi, B. J. (Eds). Oxford: Oxford University Press.

Duellman, W. E. \& Trueb, L. (1986). Biology of amphibians. New York: McGraw-Hill.

Fish, D. (1983). Phytotelmata: flora and fauna. In Phytotelmata: terrestrial plants as hosts for aquatic insect communities: 101-128. Frank, J. H. \& Lounibos, L. P. (Eds). Medford, New Jersey: Plexus Publishing.

Gosner, K. L. (1960). A simplified table for staging anuran embryos and larvae with notes on identification. Herpetologica 18: $183-190$.

Jungfer, K. H. (1996). Reproduction and parental care of the coronated treefrog, Anotheca spinosa (Steindachner, 1864) (Anura: Hylidae). Herpetologica 52: 25-32.

Jungfer, K. H. \& Weygoldt, P. (1999). Biparental care in the tadpole-feeding Amazonian treefrog Osteocephalus oophagus. Amphibia-Reptilia 20: 235-249.

Kam, Y.-C., Chuang, Z.-S. \& Yen, C.-F. (1996). Reproduction, oviposition-site selection and larval oophagy of an aboreal nester, Chirixalus eiffingeri (Rhacophoridae), from Taiwan. $J$. Herpetol. 30: 52-59.

Kam, Y.-C., Chen, Y.-H., Chen, T.-C. \& Tsai, I.-R. (2000). Maternal brood care of an arboreal breeder, Chirixalus eiffingeri (Anura: Rhacophoridae) from Taiwan. Behaviour 137:137-152.

Kam, Y.-C., Lin, C.-F., Lin, Y.-S. \& Tsai, Y.-F. (1998a). Density effects of oophagous tadpoles of Chirixalus eiffingeri (Anura: Rhacophoridae): importance of maternal brood care. Herpetologica 54: 425-433.

Kam, Y.-C., Yen, C.-F. \& Hsu, J.-L. (1998b). Water balance, growth, development, and survival of arboreal frog eggs (Chirixalus eiffingeri, Rhacophoridae): importance of egg distribution in bamboo stumps. Physiol. Zool. 71: 534-542.

Kuramoto, M. (1973). The amphibians of Iriomote of the Ryukyu Islands: ecological and zoogeographical notes. Bull. Fukuoka Univ. Edu. 22 (Part III): 139-151.

Laessle, A. M. (1961). A micro-limnological study of Jamaican bromeliads. Ecology 42: 499-517.

Lannoo, J. M., Townsend, D. S. \& Wassersug, R. J. (1987). Larval life in the leaves: arboreal tadpole types, with special attention to the morphology, ecology, and behaviour of the oophagous Osteopilus brunneus (Hylidae) larva. Fieldiana Zool. 38: $1-31$.
Licht, L. E. (1967). Growth inhibition in crowded tadpoles: intraspecific and interspecific effects. Ecology 48: 736-745.

Lin, C. F. (1996). Maternal reproductive investment and the population ecology of tadpoles in Chirixalus eiffingeri. M.S. Thesis, National Taiwan University, Taiwan, ROC. (In Chinese.)

Lue, K.-Y. (1990). The amphibians and reptiles of Taiwan. The Manuals of Wildlife Resources Inventory in Taiwan (2). Taipei: Council of Agriculture, Executive Yuan. (In Chinese.)

Myers, C. W., Daly, J. W. \& Martínez, V. (1984). An arboreal poison frog (Dendrobates) from western Panama. Am. Mus. Nov. 2783: 1-20.

Petranka, J. W. (1989). Chemical interference competition in tadpoles: does it occur outside laboratory aquaria? Copeia 1989: 921-930

Richards, C. M. (1958). The inhibition of growth in crowded Rana pipiens tadpoles. Physiol Zool 31: 138-151.

Richards, C. M. (1962). The control of tadpole growth by algaelike cells. Physiol Zool 35: 285-296.

SAS Institute, Inc. (1996). SAS/STAT User's guide. Gary: SAS Inst. Inc.

Savage, R. M. (1952). Ecological, physiological and anatomical observations on some species of anuran tadpoles. Proc. Zool. Soc. Lond. 22: 467-514.

Semlitsch, R. D. \& Caldwell, J. P. (1982). Effects of density on growth, metamorphosis, and survivorship in tadpoles of Scaphiopus holbrooki. Ecology 63: 905-911.

Steinwascher, K. (1978). Interference and exploitation competition among tadpoles of Rana utricularia. Ecology 59: 1039-1046.

Steinwascher, K. (1979a). Host-parasite interactions as potential population-regulating mechanism. Ecology 60: 884-890.

Steinwascher, K. (1979b). Competitive interactions among tadpoles: response to resource level. Ecology 60: 1172-1183.

Summers, K. (1999). The effects of cannibalism on Amazonian poison frog eggs and tadpole deposition and survivorship in Heliconia axil pools. Oecologia 119: 557-564.

Thompson, R. L. (1996). Larval habitat, ecology, and parental investment of Osteopilus brunneus (Hylidae). In Contributions to West Indian herpetology: a tribute to Albert Schwartz: 259-269. Powell, R. \& Henderson, R. W. (Eds). Ithaca: Society for the Study of Amphibians and Reptiles.

Ueda, H. (1986). Reproduction of Chirixalus eiffingeri (Boettger). Sci. Rep. Lab. Amphibian Biol., Hiroshima Univ. 8: 109-116.

Wassersug, R. J., Frogner, K. J. \& Inger, R. F. (1981). Adaptations for life in tree holes by rhacophorid tadpoles from Thailand. J. Herpetol. 15: 41-52.

Weygoldt, P. (1980). Complex brood care and reproductive behaviour in captive poison-arrow frogs, Dendrobates pumilio O. Schmidt. Behav. Ecol. Sociobiol. 7: 329-332.

Weygoldt, P. (1987). Evolution of parental care in dart poison frogs (Amphibian: Anura: Dendrobatidae). Z. Zool. Syst. Evolut.-forsch. 25: 61-67.

Wilbur, H. M. (1977). Density-dependent aspects of growth and metamorphosis in Bufo americanus. Ecology 58: 196-200. 